\title{
Correction to: Utility and accuracy of transient elastography in determining liver fibrosis: a case-control study
}

\author{
Vikas Jain ${ }^{1} \cdot$ Ujjal Poddar $^{1}$ (D) $\cdot$ Tajwar Singh Negi $^{2} \cdot$ Vivek A Saraswat $^{2} \cdot$ Narendra Krishnani $^{3} \cdot$ Surender Kumar Yachha $^{1}$. \\ Anshu Srivastava ${ }^{1}$
}

Published online: 26 February 2020

(C) Springer-Verlag GmbH Germany, part of Springer Nature 2020

\section{Correction to: European Journal of Pediatrics https://doi.org/10.1007/s00431-019-03561-y}

The author regrets that one of the author's name was incorrectly presented in the published version of this article. The third author's name original read as "Tajwer Singh Negi" this should have been "Tajwar Singh Negi".

The original article has been corrected.

The online version of the original article can be found at https://doi.org/ 10.1007/s00431-019-03561-y

Ujjal Poddar

ujjalpoddar@hotmail.com

Vikas Jain

v.join111@gmail.com

Tajwar Singh Negi

tsnegipgi@gmail.com

Vivek A Saraswat

profviveksaraswat@gmail.com

Narendra Krishnani

narendrakrishnani@yahoo.co.in
Surender Kumar Yachha

skyachha@yahoo.co.in

Anshu Srivastava

avanianshu@yahoo.com

1 Department of Pediatric Gastroenterology, Sanjay Gandhi Postgraduate Institute of Medical Sciences, Raebareli Road, Lucknow 226014, India

2 Department of Gastroenterology, Sanjay Gandhi Postgraduate Institute of Medical Sciences, Lucknow, India

3 Department of Pathology, Sanjay Gandhi Postgraduate Institute of Medical Sciences, Lucknow, India 